\title{
POINT-TRANSITIVE ACTIONS BY A STANDARD METRIC THREAD
}

\section{J. T. BORREGO}

1. Introduction. An action is a continuous function $\alpha: T \times X \rightarrow X$ where $T$ is a (topological) semigroup, $X$ is a Hausdorff space, and $\alpha\left(t_{1} t_{2}, x\right)=\alpha\left(t_{1}, \alpha\left(t_{2}, x\right)\right)$ for all $t_{1}, t_{2} \in T$ and $x \in X$. If $Q(\alpha)$ $=\{x \mid \alpha(T \times\{x\})=X\}$ is not empty, then $\alpha$ is called a point-transitive action. Actions by semigroups have been studied in [1], [2], [4] and [9]. The purpose of this note is to describe how the point-transitive actions of a standard metric thread may be constructed from pointtransitive actions of usual, nil, and min $I$-semigroups which have been classified in [2]. The reader is referred to [6], [8], and [10] for general information about the theory of semigroups.

A standard metric thread is a semigroup which is homeomorphic to a closed interval of real numbers and which has an identity at one endpoint and a zero at the other. This type of semigroup has been studied in [5] and [7]. A usual $I$-semigroup is a semigroup iseomorphic (topologically isomorphic) to $[0,1]$ under the usual multiplication; a nil $I$-semigroup is a semigroup iseomorphic to $[0,1] /\left[0, \frac{1}{2}\right]$; a min $I$-semigroup is a semigroup iseomorphic to $[0,1]$ under the multiplication $x y=\min (x, y)$. We shall have occasion to use the following theorem due to Mostert and Shields [7].

TheOREm 1 [7]. Let $T$ be a standard metric thread and $E$ be the set of idempotents. If $x, y \in E$ then $x y=\min (x, y)$. Let $C$ be the closure of a component of the complement of $E$ in $T$. Then $C$ is a usual I-semigroup or a nil I-semigroup. If $x \in C$ and $y \notin C$ then $x y=\min (x, y)$.

A function $f$ from a semigroup to a Hausdorff space is said to be a multiplicative function if and only if $f\left(t_{1}\right)=f\left(t_{2}\right)$ implies $f\left(t t_{1}\right)=f\left(t t_{2}\right)$ and $f\left(t_{1} t\right)=f\left(t_{2} t\right)$ for all $t$ in the semigroup. The following theorem which is proved in [1] is a valuable aid in the study of point-transitive actions by Abelian semigroups.

Theorem 2 [1]. Let $T$ be a compact, Abelian semigroup and let $X$ be a compact, Hausdorff space.

(1) If $h: T \rightarrow X$ is a multiplicative function, then $t x=h\left(t h^{-1}(x)\right)$ defines an action of $T$ on $X$.

Received by the editors February 3, 1969. 
(2) If $T \times X \rightarrow X$ is an action and $x \in Q=\{y \in X \mid T y=X\}$, then the function $h: T \rightarrow X$ defined by $h(t)=t x$ is multiplicative. Moreover, $t y=h\left(t h^{-1}(y)\right)$ for all $t \in T$ and $y \in X$.

2. Construction. We shall use the following notation through the remainder of this note. Let $T$ be a standard metric thread with zero 0 and identity $e ; E=\left\{x \in T \mid x^{2}=x\right\}$, i.e., the set of all idempotents of $T$; $\mathcal{e}=\{C \subset T \mid C$ is the closure of a component of $T \backslash E$ or $C$ is a nondegenerate interval contained in $E\} ; F=\{$ inf $C \mid C \in \mathbb{e}\}$; for each $x \in F$ let $C_{x} \in \mathcal{e}$ such that $x=\inf C_{x}$; if $x=\inf C_{x}$ then $x+1=\sup C_{x}$; and let $Z=F \cup(T \backslash \cup C) \cup\{e\}$. We shall assume an ordering $\leqq$ on $T$ defined by $t_{1} \leqq t_{2}$ if and only if $t_{1}=0$ or $t_{1}$ separates 0 from $t_{2}$.

Theorem 3. Let $g: Z \rightarrow[0,1]$ be a continuous order preserving function with $g(z)=0$ and $g(e)=1$. For each $x \in F$ let $\alpha_{x}$ be a pointtransitive action of $C_{x}$ on $[g(x), g(x+1)]$ with $g(x+1) \in Q\left(\alpha_{x}\right)$ $=\left\{z \in[g(x), g(x+1)] \mid \alpha_{x}\left(C_{x} \times\{z\}\right)=[g(x), g(x+1)]\right\}$. Define $\alpha: T$ $\times[0,1] \rightarrow[0,1]$ by

$$
\begin{aligned}
& \alpha(t, z)=\alpha_{x}(t, z) \quad \text { if } t \in C_{x} \quad \text { and } \quad g(x) \leqq z \leqq g(x+1) \text {, } \\
& =\alpha_{x}(t, x+1) \quad \text { if } t \in C_{x} \quad \text { and } \quad g(x+1)<z \text {, } \\
& =z \quad \text { if } t \in C_{x} \quad \text { and } z<g(x) \text {, } \\
& =g(t) \quad \text { if } t \in T \backslash \cup \mathcal{C} \text { and } g(t) \leqq z \text {, } \\
& =z \quad \text { if } t \in T \backslash \cup \mathcal{C} \text { and } z<g(t) \text {. }
\end{aligned}
$$

Then $\alpha$ is a point-transitive action with $1 \in Q(\alpha)$.

Proof. For $x \in F$ let $h_{x}: C_{x} \rightarrow[g(x), g(x+1)]$ be defined by $h_{x}(t)$ $=\alpha_{x}(t, g(x+1))$. Define $h: T \rightarrow[0,1]$ by

$$
\begin{aligned}
h(t) & =h_{x}(t) & & \text { if } t \in C_{x}, \\
& =g(t) & & \text { if } t \in(T \backslash \cup \mathcal{C}) .
\end{aligned}
$$

We shall show that $h$ is a continuous multiplicative function and $\alpha(t, z)=h\left(t h^{-1}(z)\right)$. The proof is divided into the following five lemmas.

Lemma 1. (1) If $x \in F$, then $h_{x}(x)=g(x)$ and $h_{x}(x+1)=g(x+1)$.

(2) Let $t_{1} \in Z, t_{2} \in T$. If $t_{1} \leqq t_{2}\left(t_{2} \leqq t_{1}\right)$, then $g\left(t_{1}\right) \leqq h\left(t_{2}\right)\left(h\left(t_{2}\right) \leqq g\left(t_{1}\right)\right)$.

Proof. (1) follows from the results in [3].

(2) If $t_{2} \in Z$, then (2) follows from the fact that $g$ is order preserving. If $t \in C_{x}$ then $h(t)=h_{x}(t) \geqq h_{x}(x)=g(x) \geqq g\left(t_{1}\right)$ since $t_{1} \leqq x$.

Lemma 2. The function $h$ is continuous. 
Proof. Suppose $\left\{t_{n}\right\}$ is a sequence which converges to $t$. It will be shown that $\left\{h\left(t_{n}\right)\right\}$ converges to $h(t)$. If there is an $x \in F$ such that $t \in$ Interior $C_{x}$, then it is clear that $\left\{h\left(t_{n}\right)\right\}$ converges to $h(t)$.

If $t \in F$, then $\left\{t_{n}\right\}$ is residually in $C_{t}$ or $C_{t-1}$, in which case $\left\{h\left(t_{n}\right)\right\}$ converges to $h(t)$ or $\left\{t_{n}\right\}$ is cofinally in $C_{t}$ or $C_{t-1}$. In the latter case, if $q$ is a cluster point of $\left\{h\left(t_{n}\right)\right\}$, then $q=h(t)$ since $h_{t-1}$ and $h_{t}$ are continuous.

Suppose $t \in(T \backslash \cup \mathcal{C})$. Let $q$ be a cluster point of $h\left(t_{n}\right)$ and $h\left(t_{n}^{\prime}\right)$ be a subsequence of $h\left(t_{n}\right)$ which converges to $q$. If $t_{n}^{\prime}$ is cofinally in $Z$, then there is a subsequence $t_{n}^{\prime \prime}$ of $t_{n}^{\prime}$ which is in $Z$. Since $\left\{t_{n}^{\prime \prime}\right\}$ converges to $t,\left\{g\left(t_{n}^{\prime \prime}\right)\right\}$ converges to $g(t)=h(t)$. Thus, we may suppose that $\left\{t_{n}^{\prime}\right\}$ is in $T \backslash Z$ so that for each $n$ there is $x_{n} \in F$ such that $t_{n}^{\prime} \in C_{x_{n}}$. It follows that $\left\{x_{n}\right\}$ and $\left\{x_{n}+1\right\}$ converge to $t$. Thus, $h\left(t_{n}^{\prime}\right)$ converges to $h(t)=g(t)$ since $g\left(x_{n}\right) \leqq h\left(t_{n}^{\prime}\right) \leqq g\left(x_{n}+1\right)$.

The remaining case, $t=e \in \mathrm{UC}$, is clear.

Lemma 3. The function $h$ is monotone.

Proof. To show $h$ is monotone it suffices to show that $h\left(t_{1}\right)=h\left(t_{2}\right)$ and $t_{1}<t<t_{2}$ implies $h(t)=h\left(t_{1}\right)=h\left(t_{2}\right)$.

Suppose $t_{1} \in C_{x_{1}}$ and $t_{2} \in C_{x_{2}}$. Since $h\left(t_{1}\right) \in\left[g\left(x_{1}\right), g\left(x_{1}+1\right)\right]$ and $h\left(t_{2}\right) \in\left[g\left(x_{2}\right), g\left(x_{2}+1\right)\right], h x_{1}$ and $h x_{2}$ are monotone [3], and $g$ is order preserving; $\quad h\left(t_{1}\right) \leqq g\left(x_{1}+1\right) \leqq g\left(t_{2}\right) \leqq h\left(t_{2}\right)$. If $\quad t \in C_{x_{1}}, \quad h\left(t_{1}\right) \leqq h(t)$ $\leqq g\left(x_{1}+1\right)$. If $x_{1}+1 \leqq t \leqq x_{2}$, then $g\left(x_{1}+1\right) \leqq h(t) \leqq g\left(x_{2}\right)$. If $t \in C_{x_{2}}$ then $g\left(x_{2}\right) \leqq h(t) \leqq h\left(t_{2}\right)$. Thus in this case $h(t)=h\left(t_{1}\right)=h\left(t_{2}\right)$.

Suppose $t_{1} \in(T \backslash \cup \mathcal{C})$ and $t_{2} \in C_{x_{2}}$. Since $t_{1} \leqq x_{2}, g\left(t_{1}\right) \leqq g\left(x_{2}\right) \leqq h\left(t_{2}\right)$. Thus, $g\left(x_{2}\right) \leqq h(t) \leqq h\left(t_{2}\right)$ for $x_{2} \leqq t \leqq t_{2}$ and $g\left(t_{1}\right) \leqq h(t) \leqq g\left(x_{2}\right)$ for $t_{1} \leqq t \leqq x_{2}$.

The other cases are similar.

Lemma 4. The function $h$ is multiplicative.

Proof. Suppose $h\left(t_{1}\right)=h\left(t_{2}\right), t_{1}<t_{2}$ and $t \in T$. The proof that $h\left(t t_{1}\right)$ $=h\left(t t_{2}\right)$ is divided into three cases.

(1) $t_{1} \leqq t \leqq t_{2}$. If $t_{1} \in E$, then $t_{1}=t t_{1} \leqq t t_{2} \leqq t_{2}$ so that $h\left(t t_{1}\right)=h\left(t t_{2}\right)$. If $t_{1} \notin E$ then $t_{1} \in C_{x_{1}}$ for some $x_{1} \in F$. Since $h\left(x_{1}+1\right)=h\left(t_{1}\right)$ and because of the results in [2], $h\left(x_{1}\right)=h\left(t_{1}\right)$. Thus, $h\left(t_{1}\right)=h\left(x_{1}\right)=h\left(t x_{1}\right) \leqq h\left(t t_{2}\right)$ $\leqq h\left(t_{2}\right)$.

(2) $t_{2}<t$. If $t_{1}, t_{2} \in C_{x}$ for some $x \in F$, then $h\left(t t_{1}\right)=h\left(t t_{2}\right)$ since $h \mid C_{x}$ is multiplicative. If $t_{1}$ and $t_{2}$ are not both in $C_{x}$ for all $x \in F$, then $t_{1}=t t_{1} \leqq t t_{2} \leqq t_{2}$ and $h\left(t t_{1}\right)=h\left(t t_{2}\right)$ by Lemma 3 .

(3) $t<t_{1}$ is similar to (2).

The following lemma concludes the proof of Theorem 3. 
Lemma 5. $\alpha(t, z)=h\left(t h^{-1}(z)\right)$ and $1 \in Q(\alpha)$.

Proof. The first statement follows from straightforward applications of Theorem 1 and the definitions of $\alpha$ and $h$.

The second statement follows from the following equalities: $1=h(e),[0,1]=h(T)=h(T e)$.

Next we shall show that the method of construction described in Theorem 3 gives all of the point-transitive actions by $T$. It is shown in [2] that if $\alpha: T \times X \rightarrow X$ is a point-transitive action, then $X$ is homeomorphic to $[0,1]$, and $Q(\alpha)=1$ and $Q(\alpha)$ is contained in the set of endpoints of $X$. Thus, without loss of generality we may assume that if $\alpha: T \times X \rightarrow X$ is a point-transitive action, then $X=[0,1]$ and $Q(\alpha)=\{1\}$.

Proposition 4. Let $\alpha: T \times[0,1] \rightarrow[0,1]$ be a point-transitive action with $1 \in Q$. Then there is a continuous order preserving function $g: Z \rightarrow[0,1]$ such that $g(z)=0$ and $g(e)=1$ and such that

$$
\alpha\left(C_{x} \times[g(x), g(x+1)]\right)=[g(x), g(x+1)]
$$

for $x \in F$. Moreover, for $x \in F, \alpha_{x}=\alpha \mid\left(C_{x} \times[g(x), g(x+1)]\right)$ is a pointtransitive action of $C_{x}$ on $[g(x), g(x+1)]$ with $g(x+1) \in Q\left(\alpha_{x}\right)$ and

$$
\begin{aligned}
& \alpha(t, z)=\alpha_{x}(t, z) \quad \text { if } t \in C_{x} \quad \text { and } \quad g(x) \leqq z \leqq g(x+1) \text {, } \\
& =\alpha_{x}(t, x+1) \quad \text { if } t \in C_{x} \quad \text { and } \quad g(x+1)<z \text {, } \\
& =z \quad \text { if } t \in C_{x} \quad \text { and } \quad z<g(x) \text {, } \\
& =g(t) \quad \text { if } t \in T \backslash \cup \mathcal{C} \text { and } g(t) \leqq z \text {, } \\
& =z \quad \text { if } t \in T \backslash \cup \mathcal{C} \text { and } z<g(t) \text {. }
\end{aligned}
$$

Proof. Let $h: T \rightarrow[0,1]$ be defined by $h(t)=\alpha(t, 1)$, and let $g=h \mid z$. Since $h(e)=1, h(z)=0$, and $h$ is monotone [3], $g$ is order preserving. Also $h\left(C_{x} \times[g(x), g(x+1)]\right)=h\left(C_{x}\right)=[g(x), g(x+1)]$. So that $\alpha_{x}$ is a point-transitive action with $g(x+1) \in Q\left(\alpha_{x}\right)$. The remainder of the proof is a straightforward application of Theorem 1.

\section{BIBLIOGRAPHY}

1. J. Aczél and A. D. Wallace, A note on generalizations of transitive systems of transformations, Colloq. Math. 17 (1967), 29-34.

2. J. T. Borrego and E. E. DeVun, Point-transitive actions by the unit interval, Canad. J. Math. (to appear).

3. H. Cohen and I. S. Krule, Continuous homomorphic images of real clans with zero Proc. Amer. Math. Soc. 10 (1959), 106-109.

4. J. M. Day and A. D. Wallace, Semigroups acting on continua, J. Austral. Math. Soc. 7 (1967), 327-340. 
5. W. M. Faucett, Compact semigroups irreducibly connected between two idempotents, Proc. Amer. Math. Soc. 6 (1955), 741-747.

6. K. H. Hofmann and P. S. Mostert, Elements of compact semigroups, Merrill, Columbus, Ohio, 1966.

7. P. S. Mostert and A. L. Shields, On the structure of semigroups on a compact manifold with boundary, Ann. of Math. (2) 65 (1957), 117-143.

8. A. B. Paalman-de Miranda, Topological semigroups, Matematisch Centrum, Amsterdam, 1964.

9. D. P. Stadtlander, Thread actions, Duke Math. J. 35 (1968), 483-490.

10. A. D. Wallace, On the structure of topological semigroups, Bull. Amer. Math. Soc. 61 (1955), 94-112.

\section{UNIVERSITY OF MASSACHUSETTS}

